\title{
EL PROFESOR DE ELE EN APUROS. EL MODO EN LAS INTERROGATIVAS SUBORDINADAS
}

\begin{abstract}
Resumen. El modo utilizado habitualmente en las interrogativas subordinadas es el indicativo. No obstante, se dan casos en los que se emplea, e incluso se admite como correcto, el subjuntivo. En el presente artículo se analizan desde el punto de vista pragmático algunas de esas excepciones modales.
\end{abstract}

Palabras clave: interrogativas subordinadas, alternancia modal, subjuntivo, indicativo.

\section{Introducción}

La inspiración para el presente artículo tiene doble origen. Por una parte, al tener la intención de rendir homenaje a nuestro maestro y guía en la lingüística, el profesor doctor Nowikow, hemos pensado que no existía mejor forma de hacerlo que centrándonos en el tema constituyente del eje principal de sus investigaciones: el sistema verbal.

Por otra parte, el tema que abordaremos en este estudio pretende ser una respuesta a las constantes dudas de nuestros alumnos acerca de las posibles alternancias modales en determinados tipos de oración.

Uno de estos casos problemáticos es el uso del modo verbal en las denominadas oraciones interrogativas indirectas. Numerosos manuales, monografías y otros trabajos de gramática española señalan el indicativo como modo típico de las interrogativas, incluidas las subordinadas (Matte Bon, 1995: 64; Sarmiento y Sánchez, 1997: 281). Se supone que algunos autores (García Santos, 1993: 191) lo hacen para no complicar demasiado el panorama verbal, principalmente en el caso de los trabajos destinados a los aprendientes de español como lengua extranjera ${ }^{1}$. No obstante, los

\footnotetext{
* Uniwersytet Jagielloński w Krakowie.

${ }^{1}$ En adelante ELE.
} 
alumnos de ELE, a medida que avanzan en su dominio lingüístico, van descubriendo que, en determinadas situaciones comunicativas, es posible emplear el modo subjuntivo en las construcciones interrogativas.

Lo confirman las gramáticas, entre ellas, ya la de Bello (1981: 678), pero, en realidad, salvo el interesante artículo de Nieuwenhuijsen (2001), son escasas las publicaciones dedicadas enteramente al tema de la selección modal en las oraciones subordinadas interrogativas. Dicha cuestión se comenta más bien «de paso» a la hora de analizar los modos verbales (Pamies y Nowikow, 2015: 64-65; Nowikow 2017: 59-60; Togeby, 1953/1975: 16-17; Borrego, 2013: 99-119). Por ello, en el presente artículo nos proponemos realizar un repaso de la información sobre la selección modal en determinado tipo de interrogativas subordinadas ${ }^{2}$.

Asimismo, buscaremos la respuesta a la pregunta de cuáles son las diferencias, si es que las hay, en la interpretación del mensaje conforme al modo utilizado. Nos interesarán en especial los aspectos semánticopragmáticos de las construcciones analizadas, para lo que partiremos de la noción de actos de habla de Searle (1986).

Como punto final, brindaremos un breve comentario acerca de las semejanzas y diferencias entre la lengua polaca y la española en este tipo de estructuras para averiguar si pueden influir en el correcto uso de los modos por alumnos polacos.

\section{Tipos de estructuras interrogativas subordinadas: delimitación del ámbito de estudio}

Antes de nada, es preciso señalar cierta confusión terminológica acerca de las estructuras interrogativas indirectas. Algunos (Bosque, 1982: 13-14) pueden restringir este término tan solo a las construcciones procedentes de la transformación de enunciados al estilo indirecto. Otros (Nowikow, 2017: 59-60 o Alcina y Blecua, 1987: 1118-1130) denominan indirecta a cualquier oración subordinada precedida de una palabra interrogativa, postura que adoptamos también en el presente artículo, tal y como se ha hecho en la Nueva Gramática de la Legua Española (2009: 3259). Además, utilizaremos indistintamente el nombre de las interrogativas subordinadas.

${ }^{2}$ Debido a limitaciones de espacio, no podemos comentar todas las subordinadas precedidas de una palabra interrogativa, puesto que, como se indicará más adelante, se trata de un tema muy complejo. Por la misma razón, tampoco podremos centrarnos en las interrogaciones directas, pese a que pueden presentar ciertas particularidades respecto al modo usado. 
Las mencionadas variaciones terminológicas se deben a la heterogeneidad funcional de las interrogativas subordinadas. Si bien todas comparten el rasgo formal de incluir una palabra interrogativa (qué, cómo, quién, cuándo, cuánto y cuál en el caso de preguntas parciales, o si, en las preguntas totales), pueden expresar contenidos funcionales y semántico-pragmáticos distintos.

No es posible analizarlas todas en este estudio. Por ello, no nos detendremos en las interrogativas propiamente indirectas, por la obvia razón de que el empleo de los modos en las mismas se somete a las reglas de la transformación al estilo indirecto $(1 \mathrm{a}, 1 \mathrm{~b})$.

(1a) ¿Qué has hecho con tu teléfono? $\rightarrow$ Te pregunto qué has hecho con tu teléfono.

(1b) ¿Qué hubiera hecho sin $t i ? \rightarrow$ Me preguntaste qué hubiera hecho sin ti.

Tampoco nos centraremos en las interrogativas con el subjuntivo imperfecto en el V2, intercambiable por el condicional si es verbo modal (Pamies y Nowikow, 2015: 36) (2), incluidos los enunciados de cortesía, ni en las estructuras semicondicionales, en las que el subjuntivo pluscuamperfecto se emplea como si se tratase del llamado tercer periodo condicional (3).

(2) Un amigo le aconsejó un psicoterapeuta que utilizaba la técnica Gestalt. No tenía ni la más remota idea de qué pudiera ser eso (Álamo, A.: El incendio del paraíso. Barcelona: Mondadori, 2004, /España/, CORPES, fecha de consulta: 22.08.2018).

Tuve suerte y al cabo de dos horas ella salió. Podía no haber salido de casa en todo el día o no haber vuelto hasta la noche. En ese caso no sé qué hubiera hecho (GonzálezSinde, Ángeles: El buen hijo. Barcelona: Planeta, 2013, /España/, CORPES, fecha de consulta: 22.08.2018).

Además, dejaremos para otra ocasión la interesantísima cuestión de las interrogativas con subjuntivo, empleado «en los contextos en que se expresa dependencia, o bien independencia o indiferencia» (Borrego, 2013: 117; NGLE, 2009: 1900-1901), que muestran similitud con las oraciones de relativo (Girón Alconchel, 1991: 110; Borrego et al., 1985: 114) (4):

(4) Todo depende de cómo evolucione en las próximas semanas (Hemero, apud Borrego, 2013: 117) (similar a: Todo depende del modo en que evolucione).

En cambio, nos interesan aquellas interrogativas indirectas que admiten tanto el indicativo, como el subjuntivo, y, en cuyo caso, la selección modal se rige por la semántica del verbo principal y la intención comunicativa del hablante. En cuanto al significado verbal, del conjunto de los verbos compatibles con las interrogativas propuesto por Borrego (2013: 102), todos ellos relacionados con el concepto de información, son 
importantes para el presente análisis los que expresan la solicitud de información o carencias en la misma (preguntar y sinónimos; no saber, no entender y sinónimos).

Dicha versatilidad semántica es el primer requisito para que se pueda hacer hincapié en uno u otro aspecto interpretativo: bien en la búsqueda de respuestas, bien en la falta de conocimientos. Si bien, como se verá más adelante, conforme al modo verbal empleado en la subordinada, son posibles también otras interpretaciones.

La intención indagativa del emisor o la carencia de la misma es el criterio principal para que las interrogativas puedan clasificarse en propias o aparentes, según la terminología empleada por Grzegorczykowa (2001: 130 ${ }^{3}$. Esa distinción general puede desarrollarse desde un enfoque pragmático con el fin de averiguar cómo la intención comunicativa influye en la selección modal y, por consiguiente, qué tipo de actos de habla pueden constituir enunciados con interrogativas subordinadas según se use el subjuntivo o el indicativo.

\section{Modo verbal vs. intención comunicativa}

\subsection{Indicativo}

Si la estructura interrogativa subordinada persigue la finalidad de conseguir información constituye un acto de habla directivo. En ese caso, el modo seleccionado es el indicativo, tal y como ocurre habitualmente en las preguntas formuladas de forma directa (5).

(5) - No estoy seguro... Sólo necesito saber si tiene muchas multas (Rojo, Alfonso: Matar para vivir. Barcelona: Plaza y Janés, 2002, /España/, CORPES, fecha de consulta: 20.10.2018).

En ocasiones, es posible que el emisor del mensaje en vez de buscar respuestas tenga simplemente la intención de comunicar sus carencias informativas, realizando de esta forma un acto de habla asertivo (6). La

${ }^{3}$ En relación con las interrogativas subordinadas Girón Alconchel (1991: 105-107) aplica una distinción parecida clasificándolas en indirectas (con modalidad interrogativa de la enunciación), o modales (en las que el emisor tan solo pretende evaluar su propio enunciado). 
elección natural en tal situación sigue siendo el indicativo, denominado por Nowikow (2017: 18) el modo de aserción.

(6) No me gusta que uses el plural, no sé quién eres y no me interesa conocerte, adiós -el perro amarillo salta sobre la puerta (Aridjis, H.: La zona del silencio. México D. F.: Punto de Lectura, 2005, /México/, CORPES, fecha de consulta: 12.10.2018).

Además, la estructura interrogativa en cuestión no pierde su potencial valor indagativo, por el hecho de que, como dice Nowikow (2017: 60), en las interrogativas parciales con el indicativo «la pregunta se focaliza en uno de los elementos de la cláusula subordinada», y en las totales «el hablante centra su mensaje en el desconocimiento del hecho».

Ambos actos de habla se pueden distinguir a partir de los factores contextuales, que precisan si se trata de un mensaje con sentido de: esta información, la necesito (acto directivo), o más bien interpretado como: esta información, no la tengo (acto asertivo).

\subsection{Subjuntivo}

No obstante lo anterior, entre las interrogativas indirectas con valor de acto de habla directivo o asertivo, se puede encontrar ejemplos con subjuntivo, si bien, se trata de una característica diatópica (7), extendida principalmente en México y Colombia (Pamies y Nowikow, 2015: 64), pero también en Centroamérica, Chile y las áreas caribeña y andina (NGLE, 2009: 1901) $)^{4}$.

Si llega a realizarse el plan como lo tenemos previsto por la noche, esto daría pie a que se pensara que el crimen fue una equivocación, un error; que no existió ningún propósito de matar al cardenal. Por eso acepto y doy mi voto a favor del plan de Adolfo. No sé qué digan ustedes (Hernández Rodríguez, R: La muerte de un cardenal. Guadalajara: Servicios editoriales, 2001, /México/, CORPES, fecha de consulta: 9.08.2018).

Esta preferencia modal americana, analizada en varios trabajos ${ }^{5}$ procede del uso común del subjuntivo en el español antiguo (NGLE, 2009: 1901; Suñer, 1999: 2185). En el español estándar actual dicho empleo del

${ }^{4}$ De hecho, el subjuntivo en las variantes americanas no es infrecuente en interrogativas directas: ¿Dónde crees que estén? (cap. 1, 25'-26', serie de RTVE La fugitiva).

${ }^{5}$ Véase: Nowikow, Álvarez García et al., 2001 «Alternancia de los modos indicativo y subjuntivo en las cláusulas completivas en el español de Puebla: aproximación sociolingüística», en A. Veiga, M. González Pereira, M. Souto Gómez (eds.), De lenguas y lenguajes, Colección Lingüística 2, Noia: Ed. Toxosoutos, 141-159; Nieuwenhuijsen, 2001; Lope Blanch, Estudios sobre el español de México, 1972. 
subjuntivo se considera arcaico y se utiliza solo con fines estilísticos. Según la NGLE (2009: 1901) «se observa un marcado decrecimiento de la frecuencia de estas construcciones en el español europeo aunque se atestiguan esporádicamente en la lengua de los dos últimos siglos».

El uso de estas construcciones se mantiene de forma simbólica en Galicia o Asturias (Suñer, 1999: 2185) o en expresiones fosilizadas (8) (Pamies y Nowikow, 2015: 64).

(8) No sé qué haga (equivalente al enunciado No sé qué hacer, habitual en el español peninsular $)^{6}$.

Además de sus empleos diatópicos o arcaizantes, el subjuntivo puede usarse en las interrogativas indirectas con el fin de aportar determinados valores semántico-pragmáticos. Se aprovecha el subjuntivo, como modo que, conforme a lo que afirma González Calvo (1995: 195), cuando hay alternancia, «siempre ofrece una mayor valoración apreciativa» $\mathrm{y}$ «tiene mayor capacidad modal o actitudinal». En tales casos, el enunciado se convierte en un acto de habla expresivo, en el que el emisor del mensaje puede resaltar diversos grados de inseguridad acerca de los hechos referidos en la subordinada.

Así pues, la RAE (apud Contreras, 1993: 65) comenta la existencia de las llamadas interrogativas dubitativas ${ }^{7}$, en las que, como el mismo nombre indica, el emisor expresa con subjuntivo su duda acerca del contenido de la pregunta. Bello (1981: 679) precisa que, si se utiliza el subjuntivo haciendo referencia al futuro y, además, «el agente de los dos verbos, subordinante y subordinado es o puede ser uno mismo», como en No sé si salga, la decisión está sujeta al arbitrio del agente, a diferencia del enunciado No sé si saldré, en el que la decisión no depende de la voluntad del emisor. Togeby (1953: 16) habla en este caso de «dilemme interne» y Borrego et al. (1985: 112) denomina deliberativas a dicho subtipo de interrogativas indirectas con subjuntivo, acentuando que el hablante efectúa una valoración de los pros y los contras acerca de la acción expresada por el verbo en subjuntivo.

No obstante, en el español moderno se considera «très rare» (Togeby, 1953: 16) el uso del subjuntivo con el mencionado valor dubitativo o deliberativo en las interrogaciones indirectas. Quizás porque el uso de dicho modo se puede neutralizar en gran parte con otras formas: en la versión dubitativa se suele utilizar el futuro (véase el ejemplo de

6 Véase: Nieuwenhuijsen (2001: 348), Suñer (1999: 2185), Borrego et al. (1985: 112-113).

7 Gili Gaya (1994: 47) utiliza el término dubitativas para referirse a las interrogativas directas totales (llamadas por el autor también generales), a las que se espera la respuesta sí o no, con el fin de distinguirlas de las parciales (determinativas). 
Nowikow, 2017: 60) (9a) y, en la deliberativa, con el infinitivo (véase el ejemplo de Borrego et al., 1985: 113) (9b).

(9a) No sé si tenga una casa. vs. No sé si tendrá una casa.

(9b) No sé qué haga. vs. No sé qué hacer.

Parece mucho más imprescindible el subjuntivo utilizado en las interrogativas subordinadas con referencia a hechos anteriores al momento de la enunciación, como ocurre en la clásica ya oración (10a) de Gili Gaya (1994: 136)

(10a) No sé en qué haya consistido mi fracaso.

De igual manera, expresa «incertidumbre del juicio» (Gili Gaya, 1994: 136), pero no de la misma forma que en las interrogativas indirectas deliberativas o dubitativas. Compárese el uso del subjuntivo (10a) con el de indicativo (10b) en la misma oración:

(10b) No sé en qué ha consistido mi fracaso.

Así, mientras que el indicativo expresa simplemente falta de conocimientos acerca de los motivos del fracaso, el subjuntivo ya no se limita a señalar una mayor inseguridad acerca de tales motivos, sino que pone en tela de juicio la pregunta formulada, debido a las dudas del emisor relativas a la veracidad del hecho comentado:

No sé en qué haya consistido mi fracaso (si es que ha habido algún fracasopersonalmente lo dudo).

frente a

No sé en qué ha consistido mi fracaso (asumo el fracaso y ahora quiero que se me den los detalles al respecto).

En tales situaciones, se resalta el valor del subjuntivo como modo de no aserción (Nowikow, 2017: 18), suspensión de afirmación (Togeby, 1953: 118) o, incluso, ficción (Gómez Torrego, 2002: 142) para que el emisor pueda distanciarse del contenido de la pregunta formulada, por considerarlo sin fundamento.

Nowikow (2017: 60) ${ }^{9}$ al comentar el enunciado (11) afirma algo parecido: el subjuntivo se emplea para cuestionar la veracidad del contenido de la pregunta. Y pone su propio ejemplo:

\footnotetext{
8 Véase también el punto de vista de Nieuwenhuijsen (2001: 354-355).

9 Véase también otro ejemplo en Nowikow (2010, en línea): «Hay casos en que los modos pueden focalizar la negación o remitir al contenido de un texto anterior. Por ejemplo, en:
} 
(11) No sé de dónde haya podido sacar nuestro crítico estas extrañas estadísticas [...].

con la interpretación de: No sé de dónde haya podido sacar estas estadísticas (si es que existen tales estadísticas, personalmente tengo mis dudas).

Mientras que en los enunciados relativos a hechos o acciones anteriores el cambio de significado que se produce con el empleo del subjuntivo es evidente, hay circunstancias contextuales en las que la intención del emisor de cuestionar la pregunta podría leerse en enunciados con referencia a hechos actuales o posteriores, pero ya no de forma tan contundente. Este podría ser el caso de la oración procedente de un cuento de Juan Marsé (12) sobre un escritor en proceso de desaparición, presentada por Nieuwenhuijsen (2001: 354):

\section{Al parecer falta un contertulio, pero no sabemos quién pueda ser.}

Aunque no se puede excluir que dicho enunciado tenga la interpretación de inseguridad acerca de la identidad del contertulio, debido al contexto situacional es posible la lectura de Se dice que falta un contertulio, si es que existe tal persona, lo cual parece dudoso ${ }^{10}$, tal y como sugiere la autora del artículo con dicho ejemplo.

Por último, en ocasiones, la pregunta formulada mediante el subjuntivo en estructuras interrogativas subordinadas puede adquirir valores mucho más expresivos que el de incertidumbre ante la veracidad del contenido indagativo. En dicha situación, el emisor intenta transmitir su asombro frente a los hechos referidos en la pregunta. El acto de habla expresivo realizado en tales enunciados abarca acontecimientos actuales y pasados, cuya existencia, a pesar de ser cierta, le parece al emisor difícil de aceptar, por ello los presenta como imposibles.

- Te has equivocado.

- No sé en qué me he/haya equivocado.

el uso del IND significa que el hablante reconoce que se ha equivocado, pero no sabe en qué. En cambio, el empleo del SUBJ focaliza la negación más bien en el contenido de la subordinada, lo que quiere decir que se cuestiona la veracidad de éste (el hecho de haberse equivocado no es seguro y es posible que no se haya producido)».

${ }^{10}$ La misma ambigüedad interpretativa se da en la oración de Ortega, citada por Togeby (1953: 17): No conviene preguntarse ahora cuál sea el origen de nuestras creencias. Borrego et al. (1985: 114) proponen interpretar dicho enunciado como una interrogación deliberativa, en la que se hace hincapié en los pros y los contras de la cuestión a la que se refiere la pregunta. No obstante, basándose en el análisis del pensamiento orteguiano realizado por Mellizo (1998: 166), se podría buscar la interpretación de cuestionar la necesidad de formular la pregunta, debido a que las creencias, según Ortega, son algo innato en el ser humano, léase, no tiene sentido buscar su origen, de ahí el subjuntivo en el V2. 
Este efecto de incredulidad ante la realización de los hechos se puede reforzar con el verbo modal poder conjugado en subjuntivo y precedido de una palabra interrogativa, como en el ejemplo 13.

(13) Pues, por ejemplo, no entiendo cómo en un país supuestamente civilizado, moderno y cabal, se pueda consentir, sin ira por parte de la Administración Sanitaria, que se desabastezcan estratégicamente las farmacias [...] («Nuestra Señora del Morro, Patrona de la Administración El Tuerto», Revista Medicina General. Madrid: Sanidad y Ediciones, 2003.03.03 /España/ (CORPES, fecha de consulta: 20.09.2018).

\subsection{Perspectiva de ELE}

Tras este breve repaso del uso del subjuntivo en las interrogativas subordinadas, cabe preguntarse cómo manejan la selección modal los aprendientes de ELE, y, en particular, los nativos de polaco. Curiosamente, a pesar de que habitualmente se recomienda a los extranjeros optar por el indicativo en este tipo de construcciones, los aprendientes polacos tienden a utilizar el subjuntivo, si bien, no suelen hacerlo de forma consciente, es decir con finalidades pragmáticas, ni tampoco por seguir las pautas del español americano. Su elección se debe, más bien, al establecimiento de analogías gramaticales, no siempre acertadas, que pueden inducir a error. En primer lugar, optan por el subjuntivo debido a la similitud de las interrogativas indirectas con otras oraciones sustantivas en forma negativa, en las que el V1 es un verbo de percepción, opinión o comunicación y, en principio, es obligatorio emplear el subjuntivo.

No veo que sea difícil. vs No veo por qué sea difícil. (¿?)

En segundo lugar, los aprendientes utilizan erróneamente el subjuntivo en las oraciones interrogativas con cuándo al confundirlas con las oraciones temporales referentes al futuro, en las que se emplea dicho modo.

Cuando se celebre la boda tendrá 28 años. vs. No sé cuándo se celebre la boda. (¿?)

Asimismo, los alumnos polacos se fundamentan en cierta convergencia semántico-funcional ${ }^{11}$ entre el condicional de su idioma materno y el subjuntivo español, modo inexistente en la lengua polaca, lo cual no siempre da resultado correcto.

${ }^{11}$ Este y otros temas se exponen de forma exhaustiva en el ámbito de la gramática contrastiva en Los modos verbales en español y en polaco (Pamies y Nowikow, 2015) y en Gramática contrastiva español-polaco (Nowikow, 2017). 
No sabemos qué horas sean las más convenientes. (¿?) vs. Nie wiemy, jakie godziny bytyby najodpowiedniejsze.

Sin embargo, otras analogías sí que podrían ayudar a comprender la alternancia modal en las interrogativas indirectas. En este sentido, los aprendientes de ELE podrían inspirarse en los valores de inseguridad o duda de las construcciones con tal vez y similares, en las que, además de la semántica inherente a la expresión principal (tal vez = 'posibilidad'), el grado de seguridad se debilita con el empleo del subjuntivo, en comparación con el mismo enunciado con indicativo.

A su vez, las construcciones en las que se cuestiona la veracidad podrían compararse con determinados recursos polacos con contenidos parecidos. Así, para traducir al polaco el ejemplo 13, procedente de Gili Gaya, tendríamos que emplear en la oración subordinada el verbo modal mieć en pasado para expresar intención y no realización, y la partícula niby, que, según Nowy słownik poprawnej polszczyzny (1999: 505), debilita la literalidad de la palabra a la que se refiere, en nuestro caso, polegać (esp. consistir), dotándola de un matiz de falsedad. Gracias a los mencionados elementos, conseguimos el mismo valor de puesta en duda del contenido expresado por el V2:

Nie wiem, na czym niby miała polegać moja porażka?

\section{Conclusiones}

Los aprendientes de ELE pueden llegar a emplear de forma correcta las estructuras interrogativas subordinadas, además de expresar contenidos adicionales, gracias a la pertinente selección modal. En primer lugar, tienen que recordar que las estructuras interrogativas indirectas admiten tanto el indicativo (más habitual), como el subjuntivo (con poca frecuencia y solo en determinados casos). La selección modal depende del tipo de acto de habla realizado mediante la interrogación.

En las construcciones interrogativas orientadas a la consecución de información, esto es, en actos de habla directivos, debería emplearse el indicativo. Si bien, no se puede olvidar que, en algunos países de habla hispana, principalmente en México, en las oraciones negativas interrogativas subordinadas se mantiene, en ocasiones, la regla del español antiguo de emplear el subjuntivo, tanto en las preguntas propias totales, como en las 
parciales. Dicho uso del subjuntivo arcaico se puede encontrar también en determinadas expresiones de algunas zonas de España.

A su vez, en ocasiones, se admite el subjuntivo en las interrogativas subordinadas, cuando la intención del hablante se limita a expresar diferentes grados de inseguridad acerca del contenido de la pregunta, $y$, en consecuencia, se trata de un acto de habla expresivo. A diferencia de los mismos enunciados con indicativo, con los que se buscan respuestas (acto directivo) o se constata algo (acto asertivo), el empleo del subjuntivo, en combinación con la palabra interrogativa, reorienta la interrogativa hacia un matiz dubitativo o deliberativo.

En algunos actos expresivos, el subjuntivo no solo puede introducir matices nuevos, sino que, incluso, puede cambiar el significado del enunciado en comparación con la misma estructura con indicativo. En tales casos el subjuntivo pone en duda la necesidad de formular la pregunta, cuestionando la veracidad de los hechos referidos en la misma.

Además, se dan casos en los que la estructura interrogativa subordinada con el verbo modal poder en subjuntivo incrementa el valor emocional del enunciado, al expresar la incredulidad ante los hechos referidos en el mismo.

Esta distinción entre los actos de habla asertivos, directivos y expresivos es crucial a la hora de seleccionar el pertinente modo verbal en las estructuras interrogativas subordinadas. En nuestra opinión, para los aprendientes ${ }^{12}$ de ELE tal clasificación es más eficaz en la selección modal que las posibles analogías con otros fenómenos gramaticales o el sistema verbal del idioma nativo.

Por tanto, en el proceso de aprendizaje, siempre en los niveles de dominio lingüístico más avanzados, es preciso resaltar el valor pragmático de las construcciones con subjuntivo en las interrogativas subordinadas, sin olvidar subrayar su uso limitado.

\section{Bibliografía}

ALCINA FRANCH, J. y BLECUA, J. M. (1987). Gramática española. Barcelona: Ariel.

BELLO, A. (1981). Gramática de la lengua castellana destinada al uso de los americanos ed. crítica de Ramón Trujillo. Santa Cruz de Tenerife: Instituto Universitario de Lingüística Andrés Bello: Cabildo Insular de Tenerife.

12 Véase NGLE (2009: 1902). 
BORREGO NIETO, J. (dir.) (2013). Gramática de referencia para la enseñanza de español. La combinación de oraciones. Salamanca: Ediciones de Salamanca.

BORREGO, J., ASENCIO, J. G. y PRIETO, E. (1985). El subjuntivo. Valores y usos. Madrid: SGEL, S.A.

BOSQUE, I. (1982). «Sobre la interrogación indirecta», Dicenda. Cuadernos de Filología Hispánica, N 1, 13-34.

CONTRERAS, L. (1993). «Si» en oraciones y cláusulas interrogativas indagativas», Boletín de Filología, 34 (1), 63-82.

GARCÍA SANTOS, J. F. (1993). Sintaxis del español. Nivel de perfeccionamiento. Madrid: Universidad de Salamanca y Santillana S.A.

GILI GAYA, S. (1994). Curso superior de sintaxis española. Barcelona: Biblograf.

GIRÓN ALCONCHEL, J. L. (1991). «Las oraciones interrogativas indirectas en la lengua culta hablada en Madrid», Revista de Filología, 7, 105-164.

GONZÁLEZ CALVO, J. M. (1995). «Sobre el modo verbal en español», Anuario de estudios filológicos, vol. 18, 177-204.

GÓMEZ TORREGO, L. (2002). Gramática didáctica del español. Madrid: Ediciones SM.

GRZEGORCZYKOWA, R. (2001). Wprowadzenie do semantyki językoznawczej. Warszawa: PWN.

MARKOWSKI, A. (1999). Nowy słownik poprawnej polszczyzny. Warszawa: PWN.

MATTE BON, F. (1995). Gramática comunicativa del español. Madrid: Edelsa.

MELLIZO, C. (1998). «Creencia orteguiana y Belief humeana», en J. DE SALAS y F. MARTÍN (compiladores), David Hume. Perspectivas sobre su obra. Madrid: Editorial Complutense, S.A., 148-165.

NGLE - Real Academia Española y Asociación de Academias de la Lengua Española (2009). Nueva gramática de la lengua española. Madrid: Espasa Libros.

NIEUWENHUIJSEN, D. (2001). «Modo verbal en las oraciones interrogativas indirectas». Nueva Revista de Filología Hispánica, XLIX (2), 339-362.

NOWIKOW, W. (2010). «Sobre la autonomía categorial del modo verbal desde una perspectiva tipológica», en G. LUQUET (ed.), Morphologie et syntaxe de l'espagnole. Méthodes d'approche. Paris: Presses Sorbonne Nouvelle, 77-87 [en línea] <https://books.openedition.org/psn/2732>, fecha de consulta: 23 de agosto de 2018.

NOWIKOW, W. (ed.) (2017). Gramática contrastiva español-polaco. Łódź: Wydawnictwo Uniwersytetu Łódzkiego.

PAMIES BERTRÁN, A. y NOWIKOW, W. (2015). Los modos verbales en español y en polaco. Łódź: Wydawnictwo Uniwersytetu Łódzkiego.

REAL ACADEMIA ESPAÑOLA: Banco de datos (CORPES XXI) Corpus del Español del Siglo XXI (CORPES) [en línea] <http://www.rae.es>, fecha de consulta: 28 de agosto de 2018.

SARMIENTO, R. y SÁNCHEZ, A. (1997). Gramática básica del español. Norma y uso. Madrid: SGEL Educación.

SEARLE, J. (1986). Actos de habla: ensayo de filosofía del lenguaje. Madrid: Cátedra. 
SUÑER, M. (1999). «La subordinación sustantiva: la interrogación indirecta», en V. DEMONTE y I. BOSQUE (eds.), Gramática descriptiva de la lengua española, vol. 2. Madrid: Real Academia Española / Espasa Calpe, 2149-2196.

TOGEBY, K. (1953/1975). Mode, aspect et temps en espagnol (Reimpresión). Kobenhavn: Ejnar Munskgaard [en línea] <http://www.royalacademy.dk/Publications/Low/614_Togeby,\%20Knud.pdf >, fecha de consulta: 21 de agosto de 2018. 